\title{
Hibridando el Aprendizaje Cooperativo, la Educación Aventura y la Gamificación a través de la carrera de orientación Hybridizing Cooperative Learning, Adventure Education, and Gamification through orienteering races \\ *Javier Lamoneda Prieto, **Sixto González-Víllora, ***Javier Fernández-Río \\ *Junta de Andalucía (España), **Universidad de Castilla-La Mancha (España), ***Universidad de Oviedo (España)
}

\begin{abstract}
Resumen. La práctica de actividad física en el medio natural no garantiza la formación de los estudiantes, ya que puede reducirse a aprendizajes técnicos o a la facilitación de sensaciones placenteras. El presente artículo aporta una propuesta de innovación educativa, cuyo objetivo es adaptar la normativa de la carrera de orientación al ámbito escolar; se promueve el desarrollo de habilidades sociales e inter-personales, así como el conocimiento, la preservación y la valoración de la riqueza del medio natural. Para ello, se ha hibridado la Educación de Aventura, por tratarse de un aprendizaje experiencial, con el Aprendizaje Cooperativo, al promover las conexiones interpersonales y la Gamificación, al aportar de forma lúdica una distribución de roles de trabajo y el empleo de nuevas tecnologías en la autoevaluación. Se muestran orientaciones prácticas para la intervención educativa tanto en la normativa (objetivos, participantes y el protocolo de inicio de prueba), en el uso de materiales y espacios (incorporando materiales autoconstruidos: educación al consumo; apps móviles: educación adecuada de las nuevas tecnologías), como en el sistema de evaluación formativo y compartido.

Palabras claves: Educación Secundaria; innovación educativa; tecnologías avanzadas; modelos pedagógicos.
\end{abstract}

\begin{abstract}
Physical activity practice in the outdoors does not guarantee students' learning, since it can be reduced to technical skills or pleasant sensations. This article presents an educative innovation proposal, which aims to adapt the rules of orienteering races to the school environment; it promotes the development of social and interpersonal skills, as well as the understanding, preservation, and appreciation of the richness of the outdoors. To achieve it, Adventure Education, experiential learning, has been hybridized with Cooperative Learning, promoting interpersonal connections, and Gamification, providing a playful distribution of roles and the use of new technologies for self-assessment. Practical guidelines are presented to guide an educational intervention both in the regulations (objectives, participants, and beginning protocol), in the use of materials and spaces (incorporating self-constructed materials: consumer education; mobile apps: appropriate instruction in new technologies), and in formative and shared assessment.
\end{abstract}

Keywords: Secondary education; educational innovation; new advanced technologies; pedagogical models.

\section{Introducción}

Una de las preocupaciones actuales es cómo hacer frente a las condiciones de vida propuestas por la sociedad postindustrial, inmersa en un mundo de nuevas tecnologías que parece quedar en el olvido el contacto con la naturaleza y el medio ambiente (Zulaika, Ros, Zuazagoitia, Echeazarra \& Lasurtegui, 2020). La práctica de actividad física en la naturaleza ha sido valorada como un recurso formativo de primer orden, que reporta beneficios en el practicante tanto en el plano físico, sociológico y formativo (Peñarrubia, Guillén \& Lapetra, 2016). En particular, la práctica del deporte de orientación ha sido considerada como facilitadora y estimulante en el plano físico e intelectual (Granero, Padilla, Cacho \& Gutiérrez, 2010), promotora de la creatividad y la originalidad de respuestas (Costa, 2001) y de la implicación global del alumnado (autonomía y asunción de responsabilidades) (Parra, 2008). Sin embargo, no toda práctica deportiva en el medio natural (MN) garantiza la formación del estudiante. Como ya expusieron Parra \& Rovira (2007), en la enseñanza de actividades físicas en el medio natural (AFMN) el docente puede caer en el peligro de convertirse en un mero inductor de sensaciones cuyo interés principal sea la enseñanza de habilidades técnicas de los diferentes deportes de aventura, sin aprovechar el potencial educativo y de construcción personal y grupal que manifiestan este tipo de prácticas. En este caso, la actividad física puede tener el riesgo de ser

Fecha recepción: 09-01-20. Fecha de aceptación: 20-03-20 Sixto González-Villora

sixto.gonzalez@uclm.es atrapada por propuestas de una sociedad de mercado que hace de la AFMN un producto más de consumo (Pérez, Delgado \& Caballero, 2010). Sin dudar del valor fisiológico que reporta las AFMN en el practicante, la Educación Física (EF) ha de aspirar a unos objetivos formativos superiores (Parra \& Rovira, 2007).

Existe un interés creciente por el estudio de prácticas innovadoras que se ajusten a las estructuras escolares del momento (Kirk, 2011). De entre los diferentes modelos pedagógicos, el aprendizaje cooperativo (AC) ha sido ampliamente reconocido y empleado con éxito en EF (Casey \& Goodyear, 2015; Dyson \& Casey, 2012; Fernández-Río, 2014; Gillies, 2014; Johnson \& Johnson, 1994). Se trata de un modelo de interés en la formación del profesorado de EF para la mejora de sus habilidades, los conocimientos para la adquisición de prácticas, la autoeficacia y el conocimiento pedagógico (Legrain, Escalié, Lafont \& Chaliès, 2018).

Por otra parte, rescatar hoy día los postulados de la educación aventura (EA) es de notoria necesidad y actualidad. A pesar de su larga tradición y reconocidos beneficios del modelo (Louw, Meyer, Strydom, Kotze \& Ellis, 2012), las carencias de una sociedad de consumo, tecnológica e individualista le devuelven protagonismo. En este sentido, aun contando la materia de EF con el bloque de contenidos de AFMN, conviene establecer pautas para garantizar que la intervención en el aula sea verdaderamente educativa, ya que es discutible que el modelo tradicional de enseñanza basado en el desarrollo de habilidades técnicas logre alcanzar las pretensiones de la EA (Parra, Caballero \& Domínguez, 2009). 
Finalmente, la microgamificación (MG; Martínez, 2017) es considerada en la presente propuesta innovadora por sentar las bases sobre las que sustentar elementos cruciales del proceso. Los elementos constituyentes de la misma: dinámicas, mecánicas y componentes (Werbach \& Hunter, 2012), facilitan el desarrollo del AC al tiempo que promueven elementos clave de la enseñanza como la diversión, la autonomía, la motivación, la experimentación o la creatividad (Llorens et al., 2016).

En base a todo lo anterior, el propósito de este trabajo es aportar modificaciones a la carrera de orientación deportiva escolar desde la hibridación de los modelos pedagógicos AC, EA y la MG, con el objetivo que los participantes no sólo desarrollen competencias propias de la orientación deportiva sino también las que aporta el modelo hibridado (tabla 1).

\section{Características de los tres modelos pedagógicos hibridados}

\section{Aprendizaje Cooperativo (AC)}

El AC es un «modelo pedagógico en el que los estudiantes aprenden con, de y por otros estudiantes a través de un planteamiento de enseñanza-aprendizaje que facilita y potencia esta interacción e interdependencia positivas y en el que docente y estudiantes actúan como co-aprendices» (Fernández-Río, 2014, p. 6). Plantea una visión integradora de las teorías de enseñanza-aprendizaje, de los objetivos de aprendizaje a largo plazo, el contexto contenido, la gestión del aula, el control del proceso y la evaluación (Metzler, 2005).

Existe unanimidad en considerar cinco variables que una actividad debe cumplir para ser considerada como cooperativa (Johnson \& Johnson, 1994): (1) interacción cara a cara: contacto directo (incluso físico) entre participantes; (2) interdependencia positiva: sólo tengo éxito si los compañeros de grupo también lo tienen (del yo al nosotros); (3) responsabilidad individual: cada integrante del grupo tiene una función que desempeñar (todos hacen algo); (4) procesamiento grupal: autoevaluación, comunicación (reflexión); y (5) habilidades interpersonales: puesta en práctica de la escucha activa, respeto al turno, felicitaciones, etc. No obstante, también se han señalado otras como: (a) participación equitativa: todos deben participar en las actividades de manera similar; y (b) igualdad de oportunidades de éxito: todos deben tener igualdad de oportunidades éxito en las tareas (Fernández-Río et al., 2018).

En un estudio de revisión reciente, Casey \& Goodyear (2015) argumentan que el AC permite desarrollar los cuatro tipos de resultados de aprendizaje relacionados con la EF: físico (mejora de habilidades y técnicas correctas), cognitivo (conocimiento táctico y toma de decisiones), social (desarrollo de habilidades interpersonales) y afectivo (motivación y compromiso). Se trata de uno de los modelos pedagógicos más ampliamente utilizados en EF internacionalmente.

\section{Educación Aventura (EA)}

El modelo de EA consiste básicamente en educar mediante experiencias directas en y a través de las AFMN y/o actividades de reto/aventura con la finalidad de contribuir al desarrollo humano tanto docente como discente (Parra et al., 2009). Sus orígenes se le atribuyen a Kurt Hahn, con la fun- dación en 1907 la United World Colleges y la escuela Gordonstoun. Estos indicios dieron paso a la escuela Outdoor Outward Bound y la National Outdoor Leadership School, que definitivamente asentaron las ideas filosóficas de Hahn. En esencia promovía tanto el desarrollo personal como la preparación de los jóvenes para la vida cívica y social.

Los principales rasgos que caracterizan a la EA pueden sintetizarse en seis aspectos (Baena, 2011; Fernández-Río et al., 2016; Fernández-Río et al., 2018; Hattie, Marsh, Neill \& Richards, 1997): (1) espacios: ofrecer actividades en lugares fuera de lo común, en ambientes desconocidos que generan cierto riesgo o en las que se hace uso creativo de espacios y materiales; (2) clima de trabajo: ambiente lúdico, de acción y aventura; (3) organización: grupos reducidos y heterogéneos; (4) actividades: que requieran de un trabajo multilateral (físico, mental, emocional y social) con una interacción frecuente e intensa para dar respuesta a barreras o problemas con respuestas divergentes; (5) duración: varias sesiones; de dos a cuatro semanas como mínimo.

El aprendizaje experiencial y el constructivismo han sido históricamente los marcos teóricos de referencia sobre los que se ha sustentado la EA (Sutherland \& Legge, 2016). Aunque la raíz del aprendizaje experiencial se debe a los trabajos de Dewey (1997), el ciclo de aprendizaje de Kolb ha sido muy utilizado (Priest \& Gass, 2005). A través de este ciclo, el participante adquiere un compromiso para desarrollar una experiencia concreta en la que pone en juego la observación y reflexión de modo que los aprendizajes son altamente transferibles a su vida real. Por su parte, el aprendizaje constructivista incorpora al modelo anterior el interés por el proceso constructivo y activo del conocimiento discente (Vigotsky, 1978). En este caso, el estudiante se convierte en participante activo, identifica y elabora cuestiones y resuelve problemas del mundo real.

Son numerosos los trabajos que muestran empíricamente los beneficios sociales de la EA. En programas de aprendizaje basados en aventura, como unidades de desafíos físicos cooperativos o el parkour en EF se encontraron mejoras en las habilidades sociales (Fernández-Río \& Suárez, 2014) o en la comprensión de las habilidades intra e interpersonales (Stuhr, Sutherland, Ressler \& Ortiz-Stuhr, 2015). Por su parte, Gibbons \& Ebbeck (2011) después de completar un programa de ocho meses de duración obtuvieron mejoras en la competencia atlética percibida, la autoestima global, la aceptación social percibida, la conducta conductual percibida y el aumento de las percepciones de respeto positivo por parte de los compañeros. En otros estudios que compararon las prácticas de EF y EA, esta lograba que la percepción social entre estudiantes fuese más real, menos plástica que en la escuela (Smith, Steel \& Gidlow, 2010). Para Tischler \& Mc Caughtry (2014), este tipo de prácticas ayudaban a reconsiderar las jerarquías sociales y diferencias de género. Recientemente se han realizado conexiones entre EA con el rendimiento académico (ciencias y matemáticas) y educación para la salud en el programa denominado «cinco-días, cinco-horas» (Finn, Yan \& McInnis, 2018).

\section{Gamificación}

El término gamificación hace referencia a aprovechar el potencial motivacional de las dinámicas de los videojuegos 
para provocar un cambio de comportamiento en diferentes contextos: negocios, rendimiento o lealtad social (Dichev \& Dicheva, 2017). En EF supone transformar la propia clase en base a una narrativa imaginaria como hilo conductor, con el fin de consolidar competencias u objetivos de aprendizaje, de promover la implicación de los participantes, empleando para ello mecánicas propias de videojuegos como retos, misiones o recompensas (Fernández, 2019; Flores, 2019). Los elementos fundamentales de la actividad gamificada pueden agruparse en tres categorías básicas (García, 2019; Werbach \& Hunter, 2012): (1) dinámicas: el tema (ambiente en el que se desarrolla la aventura), la narrativa (historia), el lugar-tiempo (escenario), la presentación, la progresión, las emociones, las limitaciones, o las relaciones, entre otras; (2) mecánicas: reglas, roles de los alumnos, desafíos, elección, competición, cooperación y/o feedback; y (3) componentes: personajes, avatares, premios, logros, trofeos, escudos, puntos, niveles, clasificaciones, etc.

En EF supone crear prácticas motrices que provoquen en los estudiantes unas expectativas recreativas de aprendizaje mediante el uso del juego como elemento esencial en el proceso (Coterón, Fernández, González \& Mora, 2017). A pesar de que se trata de un proceso global y coordinado a largo plazo, puede emplearse en cortos períodos de tiempo. A este tipo de práctica se ha denominado gamificación superficial (Martínez, 2017) o MG (Fernández-Río \& Flores, 2019). Estos últimos autores consideran que, debido a su pujanza, ya puede considerarse como un modelo pedagógico emergente al que tan solo le falta mayor número de evidencias científicas.

\section{Hacia un nuevo modelo hibrido: $A C+E A+M G$}

Las hibridaciones de modelos pedagógicos en EF parecen tener una ventaja respecto a la intervención de un modelo pedagógico aislado por varios aspectos como que al relacionar varios modelos se potencian sus beneficios educativos, se reducen algunas de las limitaciones que un modelo singular puede tener, o al combinar varios modelos se puede realizar una educación más individualizada y adaptada a cada contexto específico (González-Víllora, Evangelio, Sierra \& Fernández-Río, 2019). En este sentido, y que sirva como ejemplificación, de educación deportiva se ha combinado e hibridado con otros como: 1) de responsabilidad personal y social con contribuciones relevantes a los ámbitos social y afectivo; 2) modelos centrados en el juego con mejoras en los ámbitos físicos, cognitivos y tácticos (González-Víllora, Evangelio, Guijarro \& Rocamora, 2020). Recientemente, se ha observado que un modelo hibridado tiene una repercusión elevada en variables pedagógicas muy interesantes en la adolescencia actual como la mejora afectiva (amistad), el rendimiento (en EF) o la salud (nivel de actividad física) (Rocamora, González-Víllora, Fernández-Río \& Arias, 2019).

Con anterioridad, ya se han presentado trabajos de innovación docente con la hibridación de modelos pedagógicos, por ejemplificar algunos casos con éxito se han realizado proyectos con varios basados en la práctica: 1) hibridación entre AC, EF relacionada con la salud y el de educación deportiva, incorporando crossfit educativo (González-Víllora, Evangelio, Fernández-Río \& Peiró, 2018); 2) hibridación del estilo actitudinal, el AC y técnicas relacionadas con el e- learning como el flipped-classroom y la evaluación formativa, incluyendo contenidos innovadores como la cabuyería y el franqueamiento de obstáculos (Pérez-Pueyo et al., 2017); 3) hibridación de los modelos de educación deportiva y responsabilidad personal y social a través de un programa de kickboxing educativo (Menéndez \& Fernández-Río, 2016); 4) hibridación del modelo EA y responsabilidad en el MN o la EA y educación (Caballero, 2015; Caballero \& Delgado, 2014; Castro \& Gómez, 2016; Baena, Granero \& Ortiz, 2012; Baena \& Granero, 2013). Estos trabajos de buenas prácticas tienen relación con la presente innovación educativa, dado que se trata de hibridaciones de modelos pedagógicos, con la inclusión de contenidos novedosos como elemento motivador, dado que los alumnos no suelen conocer estos contenidos, puesto que no son de los más comunes en las programaciones de EF, y aplicando metodologías activas con rigor, lo cual permite, como ya se dijo previamente, una mayor contextualización del proceso de enseñanza-aprendizaje y la individualización en relación a los diferentes ritmos de aprendizaje.

En lo que respecta a la hibridación que se propone en este trabajo, la conexión AC-EA es relativamente reciente (Fernández-Río, 2015). En este caso, las experiencias prácticas previas publicadas no son numerosas; aun así, se encuentran algunas aportaciones interesantes como los desafíos físicos cooperativos (Fernández-Río, 1999) o el parkour (Fernández-Río \& Suárez, 2014), pero no hay propuestas pedagógicas con la orientación deportiva escolar. Tras revisar los dos modelos (AC-EA), se detecta un fuerte vínculo entre ambos. La EA emplea la cooperación entre los estudiantes como medio para promover el escultismo (educar a jóvenes en la exploración con base en valores y juego al aire libre), la iniciativa personal, el desarrollo del carácter, en definitiva, el aprendizaje de recursos para la vida en sociedad. Por esta razón, existen autores que afirman que «las actividades de aventura por su naturaleza son cooperativas» (Henton, 1996; p. 92). Es más, en ambos modelos aparece la necesidad de discutir y procesar la información entre estudiantes que ha venido denominándose «debrief» (informar). Sin embargo, para otros, esta interacción entre participantes no se produce verdaderamente hasta el momento en el que el AC es incorporado realmente en la AE (Metzler, 2005). La fusión entre AC y EA puede enriquecer a ambos. Si bien, la EA tiene como objeto la promoción de valores sociales (Schoel, Prouty, \& Radcliffe, 1988), determinadas prácticas pueden poner en entredicho alcanzar esta meta; especialmente para el desarrollo interpersonal (Louw et al., 2012).

Por lo tanto, el modelo hibridado podría lograr que en el corazón de la EA se incluyese el AC (Fernández-Río, 2015), generando así un programa de AFMN con garantías para el desarrollo integral del estudiante. Esta hibridación permitiría engendrar un modelo que incorporase el AC al MN. Esta nueva interacción podría favorecer un acercamiento a la sensibilización y aprecio por el entorno natural.

La innovación docente de esta aportación pedagógica no solo se basa en lo explicado anteriormente, sino que a la hibridación de estos dos modelos se suma la contribución de la MG, dando como resultado el modelo híbrido: $\mathrm{AC}+\mathrm{EA}+\mathrm{MG}$. La incorporación de elementos gamificados es relevante porque permite fusionar de un modo lúdico y con 
especial motivación entre los estudiantes los elementos específicos del deporte de orientación (valoración de paso por puntos de control y registro de tiempos) con aspectos de interés en la EA (educación integral y escultismo) y del AC (interacción e interdependencia positiva entre participantes), como se puede observar en la tabla 1.

Tabla 1.

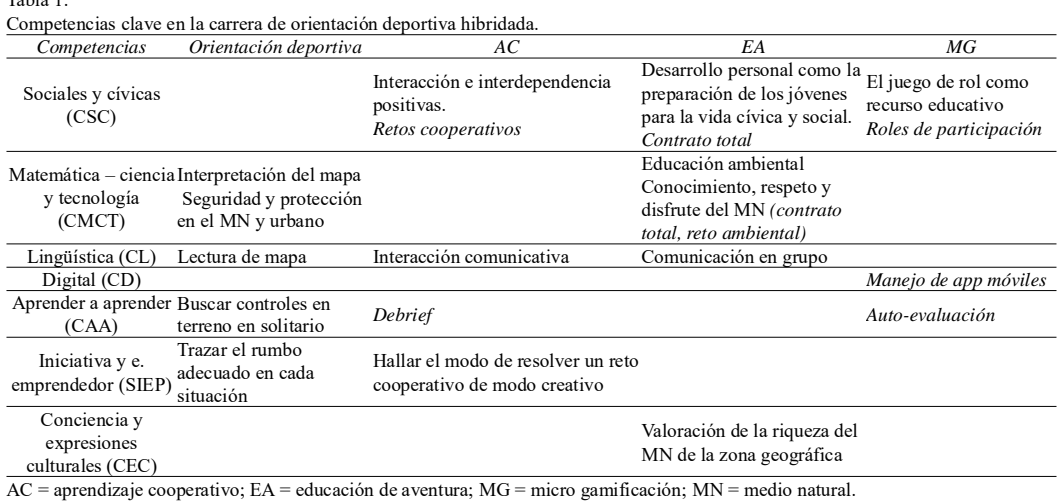

teriormente (Johnson \& Johnson, 1994): interacción cara a cara, interdependencia positiva, responsabilidad individual, procesamiento grupal y habilidades interpersonales.

La carrera de orientación educativa, siguiendo el modelo AC+EA integra cuatro zonas en las salidas: 1) organización de grupos cooperativos: previo a la cámara de llamadas se estable un tiempo para la explicación de normas de la prueba, organización de grupos, roles y entrega de material en formato Puzzle de Aronson (1978); 2) cámara de llamadas: cada grupo es nombrado para iniciar la actividad; 3 ) compromiso educativo: tras pasar por la cámara de llamadas, el grupo se sitúa en círculo, sentados, realizan una lectura de las normas del AC y adquieren un compromiso; para ello, se emplea el «dado del AC» (figuras 1 y 2) con el fin de abordar la educación en valores de un modo motivador, dinámico y lúdico;

\section{Propuesta de innovación}

\section{Normativa: objetivos y participantes}

El deporte de orientación es una disciplina individual cuya esencia es realizar un recorrido previamente diseñado por la organización, registrando el paso por los puntos de control señalados en el mapa. El participante debe tener la capacidad de leer mapas, observar la realidad y la naturaleza, analizar diferentes rutas, decidir el recorrido más favorable y ejecutar con eficacia para hallar la baliza en el terreno. Una de las principales adaptaciones de la carrera de orientación al modelo hibrido propuesto es la de pasar de ser una actividad psicomotriz a socio-motriz y, por consiguiente, incorporar como objetivo la socialización, la cooperación y la educación ambiental. La organización de los participantes deja de ser individual y pasa a estar formada por grupos reducidos y heterogéneos con roles compartidos y rotatorios (tabla 2).

\begin{tabular}{|c|c|c|c|}
\hline Nombre & Función & Rota cada baliza & Descripción \\
\hline 1 Descodificador & $\begin{array}{l}\text { Registro de código } \\
\text { (grapado o lectura } \mathrm{QR} \text { ) }\end{array}$ & Sí & $\begin{array}{l}\text { Registra el paso por baliza con } \\
\text { un grapado (pinza) en hoja de } \\
\text { control }\end{array}$ \\
\hline 2 Cabeza pensante & Mapa (guiar al grupo) & Sí & $\begin{array}{l}\text { Llevar el mapa, guiar al grupo } \\
\text { de una baliza a otra }\end{array}$ \\
\hline 3 Secretario & $\begin{array}{l}\text { Registro y evaluación } \\
\text { de retos }\end{array}$ & Sí & $\begin{array}{l}\text { Verifica que el reto se ha } \\
\text { logrado o no y valora el } \\
\text { comportamiento del grupo }\end{array}$ \\
\hline 4 Educador ambiental & $\begin{array}{l}\text { Búsqueda hojas y } \\
\text { frutos }\end{array}$ & Sí & $\begin{array}{l}\text { Clasifica las hojas en el blog de } \\
\text { ed. Ambiental }\end{array}$ \\
\hline 5 Capitán & $\begin{array}{l}\text { Coordinador principal } \\
\text { de grupo }\end{array}$ & No & $\begin{array}{l}\text { Vela por el grupo, valora el } \\
\text { trabajo cooperativo }\end{array}$ \\
\hline
\end{tabular}

\section{Protocolo de inicio de la prueba}

La salida oficial en la carrera de orientación es de tipo «contrarreloj», en la que cada corredor recibe con el mapa la tarjeta de control de forma individual con un tiempo de diferencia entre participante. En este formato, el participante recibe el material oficial (mapa), tiene un tiempo de espera (para organizar la estrategia que seguirá en la carrera) y se le asigna un tiempo oficial de salida individual. Siguiendo las características de la EA se debe incorporar el interés por crear un clima de trabajo adecuado: lúdico, de acción y aventura (Baena, 2011; Fernández-Río et al., 2016; Fernández-Río et al., 2018; Hattie, Marsh, Neill \& Richards, 1997). Por parte del AC debe cumplirse los cinco condicionantes señalados an- y 4) explicación del mapa, registro de recorrido y salida con sistema QR.

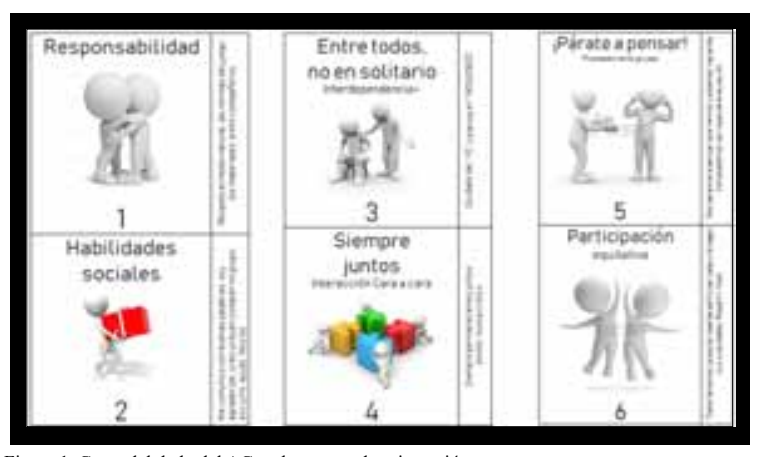

Figura 1. Caras del dado del AC en la carrera de orientación.

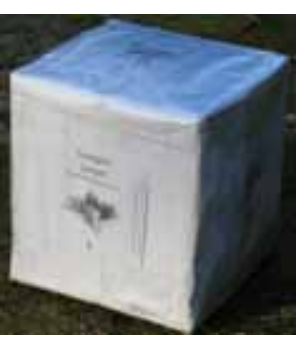

Figura 2. Dado del AC.

\section{Sistema de evaluación}

La carrera de orientación emplea como criterio de evaluación el registro de tiempos de cada corredor, la supervisión de la hoja de control para valorar que se hallaron correctamente todos los controles y la revisión de que el corredor ha respetado las normas de la prueba (no seguir a otro participante). La modificación siguiendo el modelo de EA es incorporar actividades que requieran de un trabajo multilateral (físico, mental, emocional y social) con una interacción frecuente e intensa para dar respuesta a barreras o problemas con respuestas divergentes de reeducación sensorial y ambiental (Baena, 2011; Fernández-Río et al., 2016; FernándezRío et al., 2018; Hattie, Marsh, Neill \& Richards, 1997). Del AC, se incorpora el procesamiento grupal (autoevaluaciones inter-grupo) y los retos cooperativos (ej., de aventura: slackline, de lógica: sopa de letras sobre cualidades de un grupo). El control de retos se realiza de forma autónoma en 
aquellos casos que no requieren uso de material y son sencillos (ej., situados en fila, sentarse en las rodillas del compañero); y con supervisión, en aquellos casos en los que implican riesgo (ej., tiro con arco), precisan de material (ej. transporte de globos), o la habilidad es muy compleja y/o es la primera vez que la realizan los alumnos/as (ej., uso de la brújula).

Finalmente, de la MG se propone un sistema de recompensas (Figura 3): (1) puntuación general: baliza encontrada $=10$ puntos, reto-cooperativo realizado correctamente $=20$ puntos y reto ambiental realizado: recolección y reconocimiento de hojas y frutos $=20$ puntos por objeto entregado y nombrado correctamente (hasta 100 puntos); (2) puntos de enfermedad: mal uso del teléfono móvil: fotografiarse, hablar, intercambiar información (sólo se permite escanear códigos QR) = -20 puntos; incumplir normas: seguir a otros grupos, no cooperar (ej., realizar la prueba de forma individual, actuar de forma violenta) $=-20$ puntos; $y$ (3) puntos de salud: (1) actitud cooperativa destacada (ej., ayudar, socorrer, comunicarse con cortesía, mostrar un gesto de afecto) $=$ 10 puntos; y (2) entrega de material al final (credencial de puntos, auto-evaluación de retos y debrief final) $=20$ puntos.

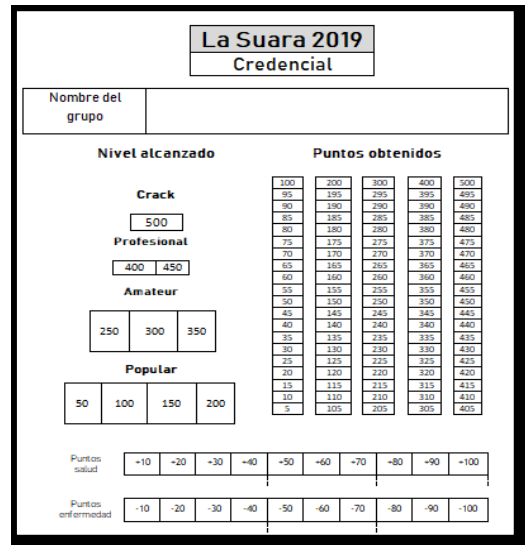

Figura 3. Sistema de puntuación en la carrera de orientación AC-EA.

\section{Materiales innovadores}

Para el óptimo desarrollo de la prueba conviene preparar con detenimiento los recursos materiales de fabricación propia, oficiales, de seguridad y tecnológicos. Las balizas pueden ser adquiridas o fabricadas de modo artesanal (figura 4); para ello los alumnos pueden colaborar, fomentando la educación adecuada al consumo. El sistema de registro de pinza es el más convencional, si bien el empleo de códigos QR facilita el cronometraje (figura 5) y favorece el aprendizaje tecnológico, potenciando la motivación de los discentes. También resulta interesante poder contar con un sistema de seguridad a bajo coste a través de candados para garantizar que las balizas permanezcan inmóviles durante la prueba (figura 6).

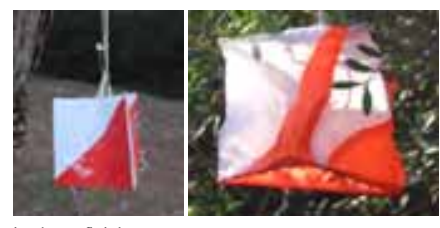

Figura 4. Baliza auto-fabricada y oficial.
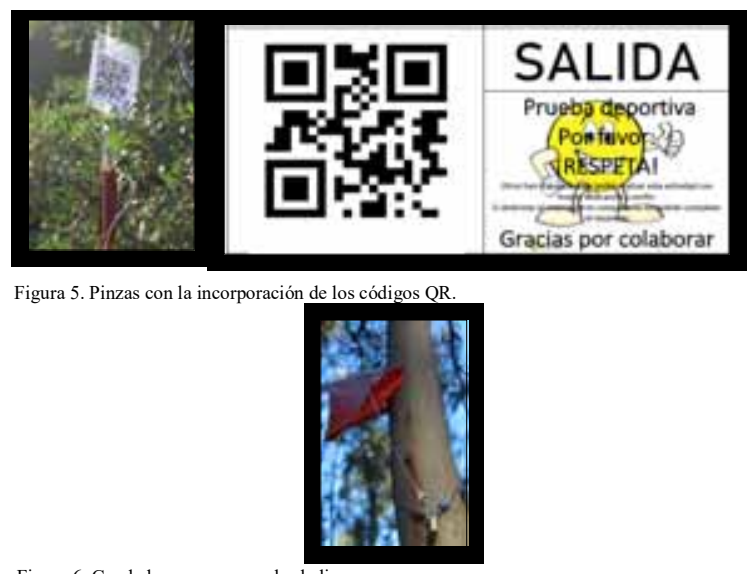

Figura 6. Candado para asegurar las balizas.

\section{Recomendaciones en el uso de las tecnologías avanza-} das

En el cronometraje de la carrera de orientación escolar resulta de interés utilizar dos apps: Bancode Scaner y dib Orienteering Dibbe, y para la identificación y conocimiento de la flora la app plantNet, como se puede observar en la figura 7. La incorporación de la utilización activa de estas apps contribuye a la familiarización y el desarrollo de los alumnos con las tecnologías avanzadas.

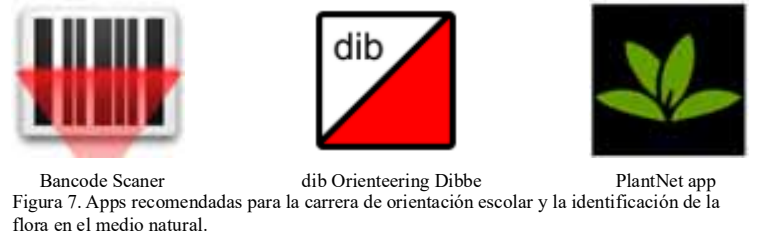

\section{Recomendaciones de uso de espacios}

Uno de los principales rasgos que caracterizan a la EA es el empleo de espacios fuera de lo común; ambientes desconocidos que generan cierto riesgo o en los que se hace un uso creativo de espacios y materiales (Baena, 2011; Fernández-Río et al., 2016; Fernández-Río et al., 2018; Hattie, Marsh, Neill \& Richards, 1997). Por ello, a pesar de poder realizar actividades de orientación en espacios urbanos o semi-urbanos conviene apostar por realizar la carrera de orientación en contacto directo con el medio natural (figura 8); lo cual, además, genera una motivación extra del alumnado.
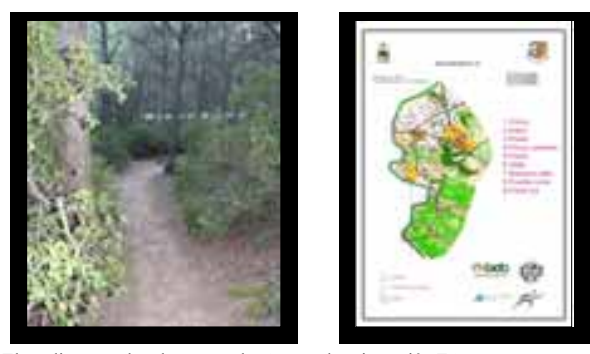

Figura 8. El medio natural y el mapa en la Carrera de orientación EA.

\section{Variantes a la normativa propuesta}

El propósito de este trabajo es aportar modificaciones en el proceso de enseñanza-aprendizaje a la carrera de orientación deportiva escolar, especialmente en el ámbito metodológico. Si bien, cada docente debe elegir según la normativa y el contexto educativos específico: nivel que muestran los alumnos/as, materiales de los que dispone y espacios de uso próximo y lejano. Las aplicaciones que pue- 
de realizar de esta propuesta didáctica pueden variar entre una sesión, una jornada escolar a una unidad didáctica completa.

En este sentido, es interesante realizar modificaciones a los diferentes tipos de disciplinas del deporte de orientación (Federación Andaluza de Deporte de Orientación, 2020) opie (diferentes distancias y/o relevos), trail-o (precisión y cronometrado), por equipos, con bicicleta de montaña, o multiaventura. Así mismo, se pueden realizar variaciones para enriquecer la enseñanza en la prueba a pie, tales como: score (búsqueda del mayor número de balizas en el menor tiempo sin orden definido de controles), seguir la línea, recorrido en estrella (recorridos de ida y vuelta desde el punto de salida) o con partes del mapa borrado.

\section{Conclusiones}

Existen pocos trabajos que aborden la iniciación al deporte de orientación, no solo a través de la enseñanza específica de la disciplina, sino también de la educación integral docente y discente. La aportación de modelos pedagógicos como la EA, el AC y la MG, como se ha mostrado en este trabajo, favorece el desarrollo de competencias de alto interés educativo que no se promueven a través de la carrera de orientación deportiva. Las principales aportaciones pedagógicas del modelo hibridado AC+EA-MG son: (1) a nivel global, la participación en grupos cooperativos, la orientación hacia la tarea y el empleo de espacios naturales; (2) durante la prueba de orientación, incluir en la salida un tiempo para la explicación de la gamificación, las normas del AC y el compromiso educativo; incorporar retos cooperativos y de aventura; y valorar el procesamiento grupal y retos superados (tabla 3 ).

Tabla 3

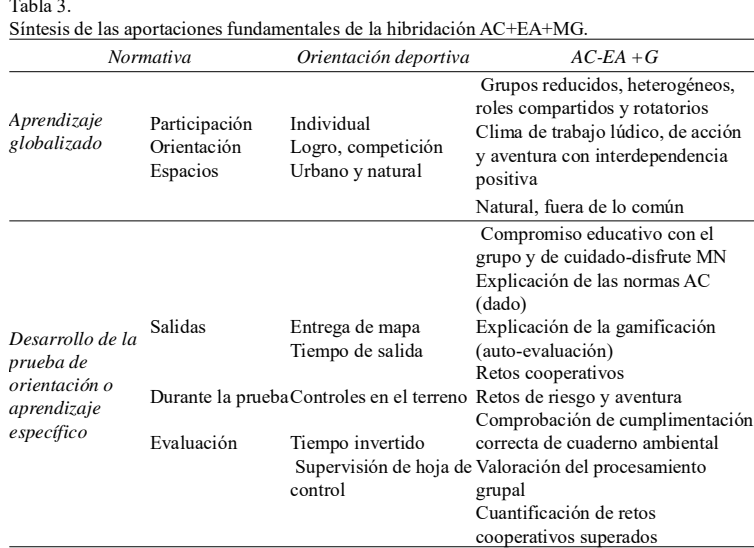

\section{Referencias}

Aronson, E. (1978). The jigsaw classroom. Beverly Hills, C. A.: Sage.

Baena, A. (2011). Programas didácticos para Educación Física a través de la Educación de Aventura. Espiral. Cuadernos del Profesorado, 4(7), 3-13.

Baena, A., Granero, A. \& Ortiz, M. (2012). Quasi-experimental study of the e ect of an adventure education programme on classroom satisfaction, physical self-concept and social goals in physical education. Psychologica Belgica, 52(4), 369-386.
Baena,A. \& Granero,A. (2013). Efecto de un programa de educación de aventura en la orientación al aprendizaje, satisfacción y autoconcepto en secundaria. Revista Iberoamericana de Diagnóstico y Evaluación Psicológica, 36, 163-182.

Caballero, P. (2015). Diseño, implementación y evaluación de un programa de actividades en la naturaleza para promover la responsabilidad personal y social en alumnos de formación profesional. Cuadernos de Psicología del Deporte, 15(2), 179-194.

Caballero, P. \& Delgado, M.A. (2014). Diseño de un programa de desarrollo positivo a través de la actividad física en el medio natural. Journal of Sport and Health Research, 6(1), 29-46.

Casey, A., \& Goodyear, V. A. (2015) Can cooperative learning achieve the four learning outcomes of physical education? A review of literature. Quest, 67(1), 56-72.

Castro, N. \& Gómez, I. (2016). Incorporación de los códigos QR en la educación física en secundaria. Retos, 29, 144-119.

Costa, P. J. (2001). Orientación en la naturaleza: manual básico de iniciación. Murcia: ASEEF

Coterón, J. González, J., Mora, C., \& Fernández-Caballero, J.(2017). Guía de iniciación a la gamificación en Educación Física. Edita: Fundación General de la Universidad Politécnica de Madrid. Recuperado de: http://afipe.es/assets/ gu $\%$ C3\%ADa -de-iniciaci $\% \mathrm{C} 3 \% \mathrm{~B} 3 \mathrm{n}$-alagamificaci $\% \mathrm{C} 3 \% \mathrm{~B} 3 n$-en-educaci $\% \mathrm{C} 3 \% \mathrm{~B} 3 \mathrm{n}$ $\mathrm{f} \% \mathrm{C} 3 \% \mathrm{ADsica}$.pdf

Dewey, J. (1997). Experience and education. New York, N. Y.: Simon \& Schuster.

Dichev, C., \& Dicheva, D. (2017). Gamifying education: what is known, what is believed and what remains uncertain: a critical review. International Journal of Educational Technology in Higher Education, 14(9), 1-36. DOI 10.1186/s412390170042-5

Federación Andaluza de deporte de Orientación (2020). Reglamento de la Federación Andaluza de deporte de Orientación. Disponible en la web (1/2/2020): https://www.web-fado.com/

Fernández-Río, J. (1999). Cooperar para adquirir las bases de una buena condición física. Élide, 1, 30-35.

Fernández-Río, J. (2014). Another step in models-based practice: Hybridizing cooperative learning and teaching for personal and social responsibility. Journal of Physical Education, Recreation and Dance, 85(7), 3-5.

Fernández-Río, J. (2015). Models-based Practice Reloaded: Connecting Cooperative Learning and Adventure Education. Journal of Physical Education, Recreation \& Dance, 86(6), 5-7. doi: 10.1080/07303084.2015.1054197.

Fernández-Río, J. (coord.) (2019). Gamificando la educación física. De la teoría a la práctica en Educación Primaria y Secundaria. Oviedo: Servicio de publicaciones de la Universidad de Oviedo.

Fernández-Río, J., Calderón, A., Hortigüela-Alcalá, D., PérezPueyo, A., \& Aznar-Cebamanos, M. (2016). Modelos pedagógicos en educación física: consideraciones teórico-prácticas para docentes. Revista Española de Educación Física y Deportes, 413, 55-75.

Fernández-Río, J., HortigüelaAlcalá, D., \& Pérez-Pueyo,A. (2018). Revisando los modelos pedagógicos en educación física. Ideas clave para incorporarlos al aula. Revista Española de Educación Física y Deportes, 423, 57-80.

Fernández-Río, J., \& Suárez, C. (2014). Feasibility and students' preliminary views on Parkour in a group of primary school children. Physical Education and Sport Pedagogy, 21(3), 
281-294. doi: 10.1080/17408989.2014.946008.

Finn, K. E., Yan, Z., \& McInnis, K. J. (2018). Promoting physical activity and science learning in an outdoor education program. Journal of Physical Education, Recreation \& Dance, 89(1),35-39. doi: 10.1080/07303084.2017.1390506.

Flores, G. (2019). ¿Jugamos al Súper Mario Bros? Descripción de una experiencia gamificada en la formación del profesorado de Educación Física. Retos, 36, 529-534.

Gibbons, S. L., \& Ebbeck, V. (2011). Team building through physical challenges in gender-segregated classes and student self-conceptions. Journal of Experiential Education, 34(1), 71-86. doi: 10.5193/JEE34.1.71.

González-Víllora, S., Evangelio, C., Fernández-Río, J., \& Peiró, C. (2018). Hibridación de tres modelos pedagógicos: aprendizaje cooperativo, educación física relacionada con la salud y educación deportiva, innovando en crossfit. En J. FernándezRío, R. Sánchez Gómez, A. Méndez-Giménez (Coords.),Actas del XI Congreso Internacional de Actividades Físicas Cooperativas (pp. 603-612).

González-Víllora, S., Evangelio, C., Sierra, J., \& Fernández-Río, J. (2019). Hybridizing pedagogical models: Asystematic review. European Physical Education Review, 25(4), 1056-1074. doi: 10.1177/1356336X18797363.

González-Víllora, S., Evangelio, C., Guijarro, E., \& Rocamora, I. (2020). Innovando con el modelo de Educación Deportiva: si buscas resultados distintos, no eduques de la misma manera. Madrid:Aula Abierta-McGraw-Hill.

Granero, A., Padilla, F. A., Cacho, M., \& Gutiérrez, H. (2010). Diferentes propuestas para la enseñanza de la orientación a nivel escolar: Orientación en el aula de educación física, orientación urbana y orientación subacuática. Apunts, 99, 34-46.

Hattie, J., Mars, H. W., Neill, J. T., \& Richards, G. E. (1997).Adventure education and outward bound: Out-of-class experiences that make a lasting difference. Review of Educational Research Spring, 67(1), 43-48.

Henton, M. (1996). Adventure in the Classroom. Dubuque, IO: Kendall/ Hunt

Johnson, D. W., \& Johnson, R. (1994). Leading the cooperative school ( $2^{\text {nd }}$ edition). Edina, NJ: Interaction Book Company.

Kirk, D. (2011). The normalization of innovation, models-based practice, and sustained curriculum renewal. Paper presented at the Association Internationale des Ecoles Superieures d'Education Physique (International Association for Physical Education in Higher Education) Conference. University of Limerick, Ireland.

Llorens, F., Gallego, F. J., Villagrá, C. J, Compañ, P., Satorre, R., \& Molina, R. (2016). Gamificación del Proceso de Aprendizaje: Lecciones Aprendidas. VAEP-RITA, 4(1), 25-32.

Louw, P. J., Meyer, C. D., Strydom, G. L., Kotze, H. N., \& Ellis, S. (2012). The impact of an adventure based experiential learning programme on the life effectiveness of black high school learners. South African Journal for Physical, Health Education, Recreation and Dance, 18(1), 55-64.

Martínez, C. M. (2017). Gamificación en Educación Física: proyecto Super Mario Bros. Publicaciones Didácticas, 89, 148152.

Menendez-Santurio, J. I., \& Fernández-Río, J. (2016). Hibridación de los modelos de Educación Deportiva y Responsabilidad Personal y Social: una experiencia a través de un programa de kickboxing educativo. Retos. Nuevas Tendencias en Educación Física, Deporte y Recreación, 30, 113-121.

Metzler, M. W. (2005). Instructional models for physical education (3rd Ed.). Scottsdale, AZ: Holcomb Hathaway.

Parra, C. (2008). Aplicaciones prácticas para piragüismo. En A. Miguel (Comp.), Actas del VI Congreso Internacional El Aula Naturaleza en la Educación Física escolar [CD-ROM]. Palencia: Patronato Municipal de Deportes.

Parra, M., \& Rovira, C. M. (2007). La tarea milagrosa. Reflexiones sobre la significabilidad socio-afectiva de las tareas. Habilidad Motriz, 28, 33-47.

Parra, M., Caballero, P., \& Domínguez, G. (2009). Pedagogía de la aventura. In $\mathrm{M}^{\mathrm{a}}$ E., García Montes (coord.), Dinámicas y estrategias de recreación, (pp. 199-260), Barcelona: Graó.

Peñarrubia, C., Guillén, R., \& Lapetra, S. (2016). Actividades en el medio natural en educación física. Cultura, Ciencia y Deporte,31(11), 27-36.

Pérez, R., Delgado, M. A., \& Caballero, P. J. (2010). Actividades físicas en el medio natural y educación ambiental es posible. Propuesta para su desarrollo. Habilidad Motriz, 35, 27-40.

Pérez-López, I. J., Rivera, E. \& Trigueros, C. (2017). La profecía de los elegidos: un ejemplo de gamificación aplicado a la docencia universitaria. Revista Internacional de Medicina y Ciencias de la Actividad Física y el Deporte, 17(66), 243260.

Pérez-Pueyo, Á., Alcalá, D. H., Álvarez, I. H., Cobo, D. V., Bernardino, C. H., García, J. G., ... \& Garijo, A. H. (2017). La hibridación de modelos pedagógicos en educación física y la evaluación formativa. Revista Infancia, Educacióny Aprendizaje, 3(2), 411-418.

Priest, S., \& Gass, M. (2005). Effective leadership in adventure programming. Champaign, IL: Human Kinetics.

Rocamora, I., González-Víllora, S., Fernández-Río, J., \&Arias, N. M. (2019). Physical activity levels, game performance and friendship goals using two different pedagogical models: Sport Education and Direct Instruction. Physical Education and Sport Pedagogy, 24(1), 87-102. doi: 10.1080/ 17408989.2018.1561839.

Schoel, J., Prouty, D., \& Radcliffe, P. (1988). Islands of healing: A guide to adventure based on counseling. Hamilton, MA: Project Adventure.

Smith, E. F., Steel, G., \& Gidlow, B. (2010). The temporary community: Student experiences of school-based outdoor education. Journal of Experiential Education, 33(2), 136150. doi: 10.5193/JEE33.2.136.

Stuhr, P. T., Sutherland, S., Ressler, J., \& Ortiz-Stuhr, E. (2015). Students' perception of relationship skills during an adventure-based learning unit within physical education. Australian Journal of Outdoor Education, 18(1), 27-38.

Sutherland, S., Stuhr, P. T., \& Ayvazo, S. (2016). Learning to teach: Pedagogical content knowledge in adventure-based learning. Physical Education and Sport Pedagogy, 21, 233-248. doi: 10.1080/17408989.2014.931365.

Tischler, A., \& McCaughtry, N. (2014). Shifting and narrowing masculinity hierarchies in physical education: Status matters. Journal of Teaching in Physical Education, 33, 342-362. doi: 10.1123/jtpe.2012-0115.

Vygotsky, L. S. (1978). Mind in society: The development of higher psychological processes. Cambridge, MA: Harvard University Press.

Werbach, K., \& Hunter, D. (2012). For the win: how game thinking can revolutionize your business. Philadelphia: Wharton Digital Press.

Zulaika L. M., Ros, I., Zuazagoitia, A., Baltar, R., Echeazarra, I., \& Ibañez, I. (2020). Propuesta de intervención en la Federación Guipuzcoana de Montaña para aumentar la participación de niños y niñas. Retos, 37, 273-283.

Retos, número 38, 2020 ( $2^{\circ}$ semestre) 